Honam Mathematical J. 34 (2012), No. 4, pp. 473-482

http://dx.doi.org/10.5831/HMJ.2012.34.4.473

\title{
INTEGRAL REPRESENTATIONS FOR SRIVASTAVA'S HYPERGEOMETRIC FUNCTION $H_{C}$
}

\author{
Junesang Choi, Anvar Hasanov and Mamasali Turaev
}

\begin{abstract}
While investigating the Lauricella's list of 14 complete second-order hypergeometric series in three variables, Srivastava noticed the existence of three additional complete triple hypergeometric series of the second order, which were denoted by $H_{A}, H_{B}$ and $H_{C}$. Each of these three triple hypergeometric functions $H_{A}$, $H_{B}$ and $H_{C}$ has been investigated extensively in many different ways including, for example, in the problem of finding their integral representations of one kind or the other. Here, in this paper, we aim at presenting further integral representations for the Srivatava's triple hypergeometric function $H_{C}$.
\end{abstract}

\section{Introduction and Preliminaries}

In the theory of hypergeometric functions of several variables, a remarkably large number of triple hypergeometric functions have been introduced and investigated. A comprehensive table of 205 distinct triple hypergeometric functions is provided in the work of Srivastava and Karlsson [17, Chapter 3]. Out of these 205 distinct triple hypergeometric functions, Lauricella [10, p. 114] introduced fourteen complete triple hypergeometric functions of the second order. He denoted his triple hypergeometric functions by the symbols $F_{1}, \ldots, F_{14}$ of which $F_{1}, F_{2}, F_{3}$ and $F_{9}$ correspond, respectively, to the three variable Lauricella functions $F_{A}^{(3)}, F_{B}^{(3)}, F_{C}^{(3)}$ and $F_{D}^{(3)}$ that are the three-variable cases of the $n$-variable Lauricella functions $F_{A}^{(n)}, F_{B}^{(n)}, F_{C}^{(n)}$ and $F_{D}^{(n)}$

Received July 2, 2012. Accepted November 30, 2012.

2010 Mathematics Subject Classification. Primary 33C20, 33C65; Secondary 33C05, 33C60, 33C70, 68Q40, 11Y35.

Key words and phrases. Multiple hypergeometric functions, Gauss hypergeometric function ${ }_{2} F_{1}$, Confluent hypergeometric functions, Eulerian integrals, Laplace integrals, Srivastava's triple hypergeometric function $H_{C}$, Exton's functions, Humbert functions, Bessel functions, Beta and Gamma functions, Appell functions, Picard's integral formula. 
(cf. [10, p. 113]; see also [1, p. 114, Equations (1) to (4)], [17, p. 33 et seq.] and [7, 8]). Saran [12] initiated a systematic study of these ten triple hypergeometric functions from Lauricella's set. Exton [6] introduced 20 distinct triple hypergeometric functions, which he denoted by $X_{1}, \ldots, X_{20}$, and investigated their twenty Laplace integral representations whose kernels include the confluent hypergeometric functions ${ }_{0} F_{1}$ and ${ }_{1} F_{1}$, and the Humbert hypergeometric functions $\Psi_{2}$ and $\Phi_{2}$ of two variables. The four Appell hypergeometric functions $F_{1}, \ldots, F_{4}$ of two variables are simply the special case of Lauricella's $n$-variable functions when $n=2$, that is,

$$
F_{1}=F_{D}^{(2)}, \quad F_{2}=F_{A}^{(2)}, \quad F_{3}=F_{B}^{(2)} \quad \text { and } \quad F_{4}=F_{C}^{(2)} .
$$

While transforming Pochhammer's double-loop contour integrals associated with the functions $F_{8}$ and $F_{14}$ (that is, $F_{G}$ and $F_{F}$, respectively) belonging to Lauricella's set of hypergeometric functions of three variables, Srivastava $[13,14]$ discovered the existence of three additional complete triple hypergeometric functions $H_{A}, H_{B}$ and $H_{C}$ of the second order, of which $H_{C}$ is defined as follows (see also [17, p. 43, Equation $1.5(12)])$ :

$$
\begin{aligned}
& H_{C}\left(a_{1}, a_{2}, a_{3} ; c ; x, y, z\right) \\
& =\sum_{m, n, p=0}^{\infty} \frac{\left(a_{1}\right)_{m+p}\left(a_{2}\right)_{m+n}\left(a_{3}\right)_{n+p}}{(c)_{m+n+p}} \frac{x^{m}}{m !} \frac{y^{n}}{n !} \frac{z^{p}}{p !} \\
& (|x|=: \mathbf{r}<1 ;|y|=: \mathbf{s}<1 ;|z|=: \mathbf{t}<1 ; \\
& \quad \mathbf{r}+\mathbf{s}+\mathbf{t}-2 \sqrt{(1-\mathbf{r})(1-\mathbf{s})(1-\mathbf{t})}<2),
\end{aligned}
$$

where, with $\mathbb{C}$ and $\mathbb{Z}_{0}^{-}$denoting the set of complex numbers and the set of nonpositive integers, respectively, $(\lambda)_{n}$ is the Pochhammer symbol defined (for $\lambda \in \mathbb{C}$ ) by

$$
(\lambda)_{n}:=\frac{\Gamma(\lambda+n)}{\Gamma(\lambda)}= \begin{cases}1 & (n=0) \\ \lambda(\lambda+1) \ldots(\lambda+n-1) & (n \in \mathbb{N}),\end{cases}
$$

$\mathbb{N}:=\{1,2,3, \ldots\}$ and $\Gamma$ being the well-known Gamma function. Of course, all 20 of Exton's triple hypergeometric functions $X_{1}, \ldots, X_{20}$ as well as Srivastava's triple hypergeometric functions $H_{A}, H_{B}$ and $H_{C}$ are included in the set of the aforementioned 205 distinct triple hypergeometric functions which were presented systematically by Srivastava and Karlsson [17, Chapter 3]. The above-stated three-dimensional region of convergence of the triple hypergeometric function in (1.1) for $H_{C}$ 
was given by Srivastava $[13,14]$ (see also Srivastava and Karlsson [17, Section 3.4]).

Various multivariable generalizations and cases of reducibility of Srivastava's functions $H_{A}, H_{B}$ and $H_{C}$ have been investigated (see, for details, [17, pp. 43-44]). Turaev [19] studied the Srivastava function $H_{A}$. Hasanov et al. [9] reproduced Srivastava's integral representations for the functions $[13,14] H_{A}, H_{B}$ and $H_{C}$. Very recently, Choi et al. ([2], [3] and [4]) also presented certain integral representations for the functions $H_{A}, H_{B}$ and $H_{C}$.

Here, in this present sequel to some of the above-mentioned works, we aim at investigating further 17 integral representations for the Srivastava function $H_{C}$, for completeness, including the five ones in [2].

\section{Integral Representations for Srivastava's hypergeometric function $H_{C}$}

Theorem. Each of the following integral representations for $H_{C}$ holds true.

$$
\begin{aligned}
& H_{C}\left(a_{1}, a_{2}, a_{3} ; c ; x, y, z\right)=\frac{\Gamma(s)}{\Gamma\left(a_{1}\right) \Gamma\left(s-a_{1}\right)} \\
& \cdot \int_{0}^{1} \xi^{a_{1}-1}(1-\xi)^{s-a_{1}-1} H_{C}\left(s, a_{2}, a_{3} ; c ; x \xi, y, z \xi\right) d \xi \\
& \quad\left(\Re(s)>\Re\left(a_{1}\right)>0\right) ; \\
& H_{C}\left(a_{1}, a_{2}, a_{3} ; c ; x, y, z\right)=\frac{\Gamma(s)}{\Gamma\left(a_{2}\right) \Gamma\left(s-a_{2}\right)} \\
& \cdot \int_{0}^{1} \xi^{a_{2}-1}(1-\xi)^{s-a_{2}-1} H_{C}\left(a_{1}, s, a_{3} ; c ; x \xi, y \xi, z\right) d \xi \\
& \quad\left(\Re(s)>\Re\left(a_{2}\right)>0\right) ; \\
& H_{C}\left(a_{1}, a_{2}, a_{3} ; c ; x, y, z\right)=\frac{\Gamma(s)}{\Gamma\left(a_{3}\right) \Gamma\left(s-a_{3}\right)} \\
& \quad \int_{0}^{1} \xi^{a_{3}-1}(1-\xi)^{s-a_{3}-1} H_{C}\left(a_{1}, a_{2}, s ; c ; x, y \xi, z \xi\right) d \xi \\
& \quad\left(\Re(s)>\Re\left(a_{3}\right)>0\right) ;
\end{aligned}
$$




$$
\begin{aligned}
& H_{C}\left(a_{1}, a_{2}, a_{3} ; c ; x, y, z\right)=\frac{\Gamma(c)}{\Gamma(c-s) \Gamma(s)} \\
& \cdot \int_{0}^{1} \xi^{s-1}(1-\xi)^{c-s-1} H_{C}\left(a_{1}, a_{2}, a_{3} ; s ; x \xi, y \xi, z \xi\right) d \xi \\
& \quad(\Re(c)>\Re(s)>0) ;
\end{aligned}
$$

$$
\begin{aligned}
& H_{C}\left(a_{1}, a_{2}, a_{3} ; c ; x, y, z\right)=\frac{\Gamma(c)}{\Gamma\left(a_{1}\right) \Gamma\left(c-a_{1}\right)} \int_{0}^{1} \xi^{a_{1}-1}(1-\xi)^{c-a_{1}-1} \\
& \cdot(1-x \xi)^{-a_{2}}(1-z \xi)^{-a_{3}} F\left(a_{2}, a_{3} ; c-a_{1} ; \frac{y(1-\xi)}{(1-x \xi)(1-z \xi)}\right) d \xi \\
& \quad\left(\Re(c)>\Re\left(a_{1}\right)>0\right) ;
\end{aligned}
$$

$$
\begin{aligned}
& H_{C}\left(a_{1}, a_{2}, a_{3} ; c ; x, y, z\right)=\frac{\Gamma(c)}{\Gamma\left(a_{2}\right) \Gamma\left(c-a_{2}\right)} \int_{0}^{1} \xi^{a_{2}-1}(1-\xi)^{c-a_{2}-1} \\
& \cdot(1-x \xi)^{-a_{1}}(1-y \xi)^{-a_{3}} F\left(a_{1}, a_{3} ; c-a_{2} ; \frac{z(1-\xi)}{(1-x \xi)(1-y \xi)}\right) d \xi \\
& \quad\left(\Re(c)>\Re\left(a_{2}\right)>0\right) ;
\end{aligned}
$$

$$
\begin{aligned}
& H_{C}\left(a_{1}, a_{2}, a_{3} ; c ; x, y, z\right)=\frac{\Gamma(c)}{\Gamma\left(a_{3}\right) \Gamma\left(c-a_{3}\right)} \int_{0}^{1} \xi^{a_{3}-1}(1-\xi)^{c-a_{3}-1} \\
& \cdot(1-y \xi)^{-a_{2}}(1-z \xi)^{-a_{1}} F\left(a_{1}, a_{2} ; c-a_{3} ; \frac{x(1-\xi)}{(1-y \xi)(1-z \xi)}\right) d \xi \\
& \quad\left(\Re(c)>\Re\left(a_{3}\right)>0\right) ;
\end{aligned}
$$

$$
\begin{aligned}
& H_{C}\left(a_{1}, a_{2}, a_{3} ; c ; x, y, z\right)=\frac{\Gamma\left(a_{1}+a_{2}\right)}{\Gamma\left(a_{1}\right) \Gamma\left(a_{2}\right)} \int_{0}^{1} \xi^{a_{1}-1}(1-\xi)^{a_{2}-1} \\
& \cdot X_{22}\left[a_{1}+a_{2}, a_{3} ; c ; x \xi(1-\xi), y(1-\xi), z \xi\right] d \xi \\
& \quad\left(\Re\left(a_{1}\right)>0 ; \Re\left(a_{2}\right)>0\right) ;
\end{aligned}
$$




$$
\begin{aligned}
& H_{C}\left(a_{1}, a_{2}, a_{3} ; c ; x, y, z\right)=\frac{\Gamma\left(a_{1}+a_{2}+a_{3}\right)}{\Gamma\left(a_{1}\right) \Gamma\left(a_{2}\right) \Gamma\left(a_{3}\right)} \\
& \cdot \int_{0}^{1} \int_{0}^{1} \xi^{a_{1}-1} \eta^{a_{1}+a_{2}-1}(1-\xi)^{a_{2}-1}(1-\eta)^{a_{3}-1} \\
& \cdot F\left[\frac{a_{1}+a_{2}+a_{3}}{2}, \frac{a_{1}+a_{2}+a_{3}+1}{2} ; c ;\right. \\
& \quad 4 \eta[x \xi(1-\xi) \eta+y(1-\xi)(1-\eta)+z \xi(1-\eta)]] d \xi d \eta \\
& \left(\Re\left(a_{1}\right)>0 ; \Re\left(a_{2}\right)>0 ; \Re\left(a_{3}\right)>0\right) ;
\end{aligned}
$$

$$
\begin{aligned}
& H_{C}\left(a_{1}, a_{2}, a_{3} ; c ; x, y, z\right)=\frac{\Gamma(c)(1+\lambda)^{a_{1}}}{\Gamma\left(a_{1}\right) \Gamma\left(c-a_{1}\right)} \int_{0}^{1} \xi^{a_{1}-1}(1-\xi)^{c-a_{1}-1} \\
& \cdot(1+\lambda \xi)^{a_{2}+a_{3}-c}[1+\lambda \xi-(1+\lambda) x \xi]^{-a_{2}}[1+\lambda \xi-(1+\lambda) z \xi]^{-a_{3}} \\
& \cdot F\left[a_{2}, a_{3} ; c-a_{1} ; \frac{y(1+\lambda \xi)(1-\xi)}{[1+\lambda \xi-(1+\lambda) x \xi][1+\lambda \xi-(1+\lambda) z \xi]}\right] d \xi \\
& \quad\left(\Re(c)>\Re\left(a_{1}\right)>0 ; \Re(\lambda)>-1\right) ;
\end{aligned}
$$

$$
\begin{aligned}
& H_{C}\left(a_{1}, a_{2}, a_{3} ; c ; x, y, z\right)=\frac{\Gamma(c)(\beta-\gamma)^{a_{1}}(\alpha-\gamma)^{c-a_{1}}}{\Gamma\left(a_{1}\right) \Gamma\left(c-a_{1}\right)(\beta-\alpha)^{c-a_{2}-a_{3}-1}} \\
& \cdot \int_{\alpha}^{\beta}(\beta-\xi)^{c-a_{1}-1}(\xi-\alpha)^{a_{1}-1}(\xi-\gamma)^{a_{2}+a_{3}-c} \\
& \cdot[(\beta-\alpha)(\xi-\gamma)-(\beta-\gamma)(\xi-\alpha) x]^{-a_{2}} \\
& \cdot[(\beta-\alpha)(\xi-\gamma)-(\beta-\gamma)(\xi-\alpha) z]^{-a_{3}} F\left(a_{2}, a_{3} ; c-a_{1} ; \sigma y\right) d \xi \\
& \quad\left(\Re(c)>\Re\left(a_{1}\right)>0 ; \gamma<\alpha<\beta\right),
\end{aligned}
$$

where

$\sigma:=\frac{(\beta-\alpha)(\alpha-\gamma)(\xi-\gamma)(\beta-\xi)}{[(\beta-\alpha)(\xi-\gamma)-(\beta-\gamma)(\xi-\alpha) x][(\beta-\alpha)(\xi-\gamma)-(\beta-\gamma)(\xi-\alpha) z]} ;$ 
$(2.12)$

$$
\begin{aligned}
& H_{C}\left(a_{1}, a_{2}, a_{3} ; c ; x, y, z\right)=\frac{\Gamma(c)(\gamma-\beta)^{a_{1}}(\gamma-\alpha)^{c-a_{1}}}{\Gamma\left(a_{1}\right) \Gamma\left(c-a_{1}\right)(\beta-\alpha)^{c-a_{2}-a_{3}-1}} \\
& \cdot \int_{\alpha}^{\beta}(\beta-\xi)^{c-a_{1}-1}(\xi-\alpha)^{a_{1}-1}(\gamma-\xi)^{a_{2}+a_{3}-c} \\
& \cdot[(\beta-\alpha)(\gamma-\xi)-(\gamma-\beta)(\xi-\alpha) x]^{-a_{2}} \\
& \cdot[(\beta-\alpha)(\gamma-\xi)-(\gamma-\beta)(\xi-\alpha) z]^{-a_{3}} F\left(a_{2}, a_{3} ; c-a_{1} ; \sigma y\right) d \xi \\
& \quad\left(\Re(c)>\Re\left(a_{1}\right)>0 ; \alpha<\beta<\gamma\right),
\end{aligned}
$$

where

$$
\sigma:=\frac{(\beta-\alpha)(\gamma-\alpha)(\beta-\xi)(\gamma-\xi)}{[(\beta-\alpha)(\gamma-\xi)-(\gamma-\beta)(\xi-\alpha) x][(\beta-\alpha)(\gamma-\xi)-(\gamma-\beta)(\xi-\alpha) z]} ;
$$

$$
\begin{aligned}
& H_{C}\left(a_{1}, a_{2}, a_{3} ; c ; x, y, z\right)=\frac{\Gamma(c)}{\Gamma\left(a_{1}\right) \Gamma\left(c-a_{1}\right)} \int_{0}^{\infty} \xi^{a_{1}-1}(1+\xi)^{a_{2}+a_{3}-c} \\
& \cdot(1+\xi-\xi x)^{-a_{2}}(1+\xi-\xi z)^{-a_{3}} F\left(a_{2}, a_{3} ; c-a_{1} ; \sigma y\right) d \xi \\
& \quad\left(\Re(c)>\Re\left(a_{1}\right)>0\right),
\end{aligned}
$$

where

$$
\sigma:=\frac{1+\xi}{(1+\xi-\xi x)(1+\xi-\xi z)}
$$

$$
\begin{aligned}
& H_{C}\left(a_{1}, a_{2}, a_{3} ; c ; x, y, z\right)=\frac{\Gamma(c)}{\Gamma\left(a_{1}\right) \Gamma\left(c-a_{1}\right)} \int_{0}^{\infty}\left(e^{-\xi}\right)^{a_{1}}\left(1-e^{-\xi}\right)^{c-a_{1}-1} \\
& \cdot\left[1-x e^{-\xi}\right]^{-a_{2}}\left[1-z e^{-\xi}\right]^{-a_{3}} F\left(a_{2}, a_{3} ; c-a_{1} ; \sigma y\right) d \xi \\
& \quad\left(\Re(c)>\Re\left(a_{1}\right)>0\right),
\end{aligned}
$$

where

$$
\sigma:=\frac{\left(1-e^{-\xi}\right) y}{\left(1-x e^{-\xi}\right)\left(1-z e^{-\xi}\right)}
$$




$$
\begin{aligned}
& H_{C}\left(a_{1}, a_{2}, a_{3} ; c ; x, y, z\right)=\frac{2 \Gamma(c)}{\Gamma\left(a_{1}\right) \Gamma\left(c-a_{1}\right)} \\
& \cdot \int_{0}^{\frac{\pi}{2}}\left(\sin ^{2} \xi\right)^{a_{1}-\frac{1}{2}}\left(\cos ^{2} \xi\right)^{c-a_{1}-\frac{1}{2}}\left[1-x \sin ^{2} \xi\right]^{-a_{2}} \\
& \cdot\left[1-z \sin ^{2} \xi\right]^{-a_{3}} F\left(a_{2}, a_{3} ; c-a_{1} ; \sigma y\right) d \xi \\
& \quad\left(\Re(c)>\Re\left(a_{1}\right)>0\right),
\end{aligned}
$$

where

$$
\sigma:=\frac{\cos ^{2} \xi}{\left(1-x \sin ^{2} \xi\right)\left(1-z \sin ^{2} \xi\right)}
$$

$$
\begin{aligned}
& H_{C}\left(a_{1}, a_{2}, a_{3} ; c ; x, y, z\right)=\frac{2 \Gamma(c)(1+\lambda)^{a_{1}}}{\Gamma\left(a_{1}\right) \Gamma\left(c-a_{1}\right)} \\
& \cdot \int_{0}^{\frac{\pi}{2}} \frac{\left(\sin ^{2} \xi\right)^{a_{1}-\frac{1}{2}}\left(\cos ^{2} \xi\right)^{c-a_{1}-\frac{1}{2}}}{\left(1+\lambda \sin ^{2} \xi\right)^{c-a_{2}-a_{3}}}\left[1+\lambda \sin ^{2} \xi-(1+\lambda) x \sin ^{2} \xi\right]^{-a_{2}} \\
& \cdot\left[1+\lambda \sin ^{2} \xi-(1+\lambda) z \sin ^{2} \xi\right]^{-a_{3}} F\left(a_{2}, a_{3} ; c-a_{1} ; \sigma y\right) d \xi \\
& \quad\left(\Re(c)>\Re\left(a_{1}\right)>0 ; \lambda>-1\right),
\end{aligned}
$$

where

$$
\sigma:=\frac{\left(1+\lambda \sin ^{2} \xi\right) \cos ^{2} \xi}{\left[1+\lambda \sin ^{2} \xi-(1+\lambda) x \sin ^{2} \xi\right]\left[1+\lambda \sin ^{2} \xi-(1+\lambda) z \sin ^{2} \xi\right]} ;
$$

$$
\begin{aligned}
& H_{C}\left(a_{1}, a_{2}, a_{3} ; c ; x, y, z\right)=\frac{2 \Gamma(c) \lambda^{a_{1}}}{\Gamma\left(a_{1}\right) \Gamma\left(c-a_{1}\right)} \\
& \cdot \int_{0}^{\frac{\pi}{2}} \frac{\left(\sin ^{2} \xi\right)^{a_{1}-\frac{1}{2}}\left(\cos ^{2} \xi\right)^{c-a_{1}-\frac{1}{2}}}{\left(\cos ^{2} \xi+\lambda \sin ^{2} \xi\right)^{c-a_{2}-a_{3}}}\left(\cos ^{2} \xi+\lambda \sin ^{2} \xi-\lambda x \sin ^{2} \xi\right)^{-a_{2}} \\
& \cdot\left(\cos ^{2} \xi+\lambda \sin ^{2} \xi-\lambda z \sin ^{2} \xi\right)^{-a_{3}} F\left(a_{2}, a_{3} ; c-a_{1} ; \sigma y\right) d \xi \\
& \quad\left(\Re(c)>\Re\left(a_{1}\right)>0 ; \lambda>0\right),
\end{aligned}
$$

where

$$
\sigma:=\frac{\left(\cos ^{2} \xi+\lambda \sin ^{2} \xi\right) \cos ^{2} \xi}{\left(\cos ^{2} \xi+\lambda \sin ^{2} \xi-\lambda x \sin ^{2} \xi\right)\left(\cos ^{2} \xi+\lambda \sin ^{2} \xi-\lambda z \sin ^{2} \xi\right)} .
$$


Here, $F$ and $X_{22}$ denote the Gaussian hypergeometric function and a new Exton type hypergeometric function, respectively:

$$
F(a, b ; c ; x)=\sum_{m=0}^{\infty} \frac{(a)_{m}(b)_{m}}{(c)_{m} m !} x^{m}
$$

and

$$
X_{22}\left(a_{1}, a_{2} ; c ; x, y, z\right)=\sum_{m, n, p=0}^{\infty} \frac{\left(a_{1}\right)_{2 m+n+p}\left(a_{2}\right)_{n+p}}{(c)_{m+n+p}} \frac{x^{m}}{m !} \frac{y^{n}}{n !} \frac{z^{p}}{p !} .
$$

Proof. There may be several methods to prove those formulas presented here (see, for example, [13] and [2]). Each of the integral representations (2.1) to (2.16) can also be proved directly by expressing the series definition of the involved special function in each integrand and changing the order of the integral sign and the summation, and finally using the following well-known relationship between the Beta function $B(\alpha, \beta)$, the Gamma function $\Gamma$ and their various associated Eulerian integrals (see, for example, [5, pp. 9-11], [15, 16, Section 1.1] and [18, p. 26 and p. 86, Problem 1]):

$$
B(\alpha, \beta)= \begin{cases}\int_{0}^{1} t^{\alpha-1}(1-t)^{\beta-1} d t & (\Re(\alpha)>0 ; \Re(\beta)>0) \\ \frac{\Gamma(\alpha) \Gamma(\beta)}{\Gamma(\alpha+\beta)} & \left(\alpha, \beta \in \mathbb{C} \backslash \mathbb{Z}_{0}^{-}\right),\end{cases}
$$

$$
\begin{aligned}
B(\alpha, \beta)=2 & \int_{0}^{\frac{\pi}{2}}(\sin \theta)^{2 \alpha-1}(\cos \theta)^{2 \beta-1} d \theta=\int_{0}^{\infty} \frac{\tau^{\alpha-1}}{(1+\tau)^{\alpha+\beta}} d \tau \\
& (\Re(\alpha)>0 ; \Re(\beta)>0)
\end{aligned}
$$

and

$$
\begin{array}{rlrl}
B(\alpha, \beta)= & \frac{(b-c)^{\alpha}(a-c)^{\beta}}{(b-a)^{\alpha+\beta-1}} \int_{a}^{b} \frac{(t-a)^{\alpha-1}(b-t)^{\beta-1}}{(t-c)^{\alpha+\beta}} d t & & (c<a<b) \\
= & (1+\lambda)^{\alpha} \int_{0}^{1} \frac{t^{\alpha-1}(1-t)^{\beta-1}}{(1+\lambda t)^{\alpha+\beta}} d t & & (\lambda>-1) \\
& (\Re(\alpha)>0 ; \Re(\beta)>0) . &
\end{array}
$$




\section{Concluding Remarks}

Integral representations for most of the special functions of mathematical physics and applied mathematics have been investigated in the existing literature. Here we have presented only some illustrative integral representations for the Srivastava's function $H_{C}$. A variety of integral representations of $H_{C}$, which may be different from those presented here, can also be provided. Integral representations (2.5), (2.9), (2.10), (2.11), (2.13) and (2.15) here include and correspond with the integral representations (4.1), (4.2), (4.3), (4.4), (4.5) and (4.6), respectively, in $[2]$.

\section{References}

[1] P. Appell and J. Kampé de Fériet, Fonctions Hypergeometriques et Hyperspheriques; Polynomes d'Hermite, Gauthier - Villars, Paris, 1926.

[2] J. Choi, A. Hasanov, H. M. Srivastava and M. Turaev, Integral representations for Srivastava's triple hypergeometric functions, Taiwanese J. Math. 15 (2011), $2751-2762$.

[3] J. Choi, A. Hasanov and M. Turaev, Integral representations for Srivastava's triple hypergeometric function $H_{A}$, Honam Math. J. 34 (2012), 113-124.

[4] J. Choi, A. Hasanov and M. Turaev, Integral representations for Srivastava's triple hypergeometric function $H_{B}$, J. Korean Soc. Math. Edu. Series B: Pure Appl. Math. 19(2) (2012), 137-145.

[5] A. Erdélyi, W. Magnus, F. Oberhettinger and F. G. Tricomi, Higher Transcendental Functions, Vol. I, McGraw-Hill Book Company, New York, Toronto and London, 1953.

[6] H. Exton, Hypergeometric functions of three variables, J. Indian Acad. Math. 4 (1982), 113-119.

[7] A. Hasanov and H. M. Srivastava, Some decomposition formulas associated with the Lauricella function $F_{A}^{(r)}$ and other multiple hypergeometric functions, Appl. Math. Lett. 19 (2006), 113-121.

[8] A. Hasanov and H. M. Srivastava, Decomposition formulas associated with the Lauricella multivariable hypergeometric functions, Comput. Math. Appl. $\mathbf{5 3}$ (2007), 1119-1128.

[9] A. Hasanov, H. M. Srivastava and M. Turaev, Decomposition formulas for some triple hypergeometric functions, J. Math. Anal. Appl. 324 (2006), 955-969.

[10] G. Lauricella, Sulle funzioni ipergeometriche a più variabili, Rend. Circ. Mat. Palermo 7 (1893), 111-158.

[11] P. A. Padnanabham, Two results on three variable hypergeometric function, Indian J. Pure Appl. Math. 30 (1999), 1107-1109.

[12] S. Saran, Hypergeometric functions of three variables, Ganita 5 (1954), 71-91.

[13] H. M. Srivastava, Hypergeometric functions of three variables, Ganita 15 (1964), 97-108. 
[14] H. M. Srivastava, Some integrals representing triple hypergeometric functions, Rend. Circ. Mat. Palermo 16 (1967), 99-115.

[15] H. M. Srivastava and J. Choi, Series Associated with the Zeta and Related Functions, Kluwer Academic Publishers, Dordrecht, Boston and London, 2001.

[16] H. M. Srivastava and J. Choi, Zeta and q-Zeta Functions and Associated Series and Integrals, Elsevier Science Publishers, Amsterdam, London and New York, 2012 .

[17] H. M. Srivastava and P. W. Karlsson, Multiple Gaussian Hypergeometric Series, Halsted Press (Ellis Horwood Limited, Chichester), John Wiley and Sons, New York, Chichester, Brisbane and Toronto, 1985.

[18] H. M. Srivastava and H. L. Manocha, A Treatise on Generating Functions, Halsted Press (Ellis Horwood Limited, Chichester), John Wiley and Sons, New York, Chichester, Brisbane and Toronto, 1984.

[19] M. Turaev, Decomposition formulas for Srivastava's hypergeometric function $H_{A}$ on Saran functions, J. Comput. Appl. Math. 233 (2009), 842-846.

Junesang Choi

Department of Mathematics, Dongguk University, Gyeongju 780-714, Korea.

E-mail: junesang@mail.dongguk.ac.kr

Anvar Hasanov

Department of Mathematics, I. M. Gubkin Russian State University of Oil and Gas,

Tashkent 100180, Uzbekistan.

E-mail: anvarhasanov@yahoo.com

Mamasali Turaev

Department of Mathematics, Dongguk University,

Gyeongju 780-714, Korea.

E-mail:mturaev@dongguk.ac.kr 